\title{
A wideband IM3 cancellation technique for CMOS attenuators
}

\author{
W. Cheng, M.S. Oude Alink, A.J. Annema, G. J.M. Wienk, B. Nauta
}

University of Twente, Enschede, Netherlands

Contact author: Wei Cheng;

Mailing address: Room 2009, Building Carre, Hallenweg 15, University of Twente, 7522NH Enschede, The Netherlands;

Telephone: office +31534892727 , mobile +31616291178 ;

Fax: +31534891034;

Email: w.cheng@utwente.nl;

\section{Abstract:}

A highly linear $\Pi$ attenuator system using a wideband IM3 cancellation technique is presented that provides 4 discrete attenuation levels with $6 \mathrm{~dB}$ spacing for DC-5GHz. For the whole band, $\mathrm{S} 11<-14 \mathrm{~dB}$, attenuation flatness $<1.6 \mathrm{~dB},+10 \mathrm{dBm}$ input $\mathrm{P}_{1 \mathrm{~dB}}$ and $+26 \mathrm{dBm}$ IIP3 are achieved. For the TV band $(0.1 \mathrm{Gz}-1.2 \mathrm{GHz})+30 \mathrm{dBm}$ IIP3 is achieved. The active area is $0.0054 \mathrm{~mm}^{2}$ in a standard 0.16um bulk CMOS process.

\section{Text:}

In the receiver path and in spectrum analyzers typically gain control blocks are used to limit the incident power to the level that the receiver circuitry can handle without degrading the linearity; in the transmitter path stringent power control is also desirable. Although variable-gain amplifiers (VGAs) traditionally implement the gain control block, attenuators based on FET transistors show superior performances on linearity, power handling capability and power consumption. 
Much effort [1-3] has been devoted to improving the linearity and power handling capability of attenuators. The adaptive bootstrapped body biasing [1] is used in a cascaded $\Pi$ attenuator to suppress the body-related parasitic effects and improve $\mathrm{P}_{1 \mathrm{~dB}}$. A $\Pi$ attenuator with parallel branches designed for discrete attenuation steps achieving $+23 \mathrm{dBm}$ IIP3 is shown in [2]. The stacked FET technique used in [3-4] distributes the signal swing among many FETs in series to reduce the drain-source voltage swing for each FET and hence reduce the IM3 distortion. However, the large transistors required by this technique bring in large parasitic capacitances, which lower the bandwidth and increase the minimum insertion loss at high frequency. Moreover, the capacitive nonlinearities introduced by large parasitic capacitances will limit the highest achievable IIP3. Therefore, this technique is mainly effective in SOI CMOS [3].

This paper proposes a wideband IM3 cancellation technique for bulk CMOS $\Pi$ attenuators where the IM3 distortion currents of transistors within the attenuator cancel each other. In the $\Pi$ attenuator in Fig. 4.3.1(upper), the voltage swing across the nonlinear output conductances of transistor $\mathrm{M}_{1}, \mathrm{M}_{2}$ and $\mathrm{M}_{3}$ generate IM3 distortion currents $i_{D}^{M_{1}}, i_{D}^{M_{2}}$ and $i_{D}^{M_{3}}$ defined from drain to source. The distortion current of $\mathrm{M}_{1}\left(i_{D}^{M_{1}}\right)$ is directed towards $\mathrm{R}_{\text {load, }}$, while the distortion currents of $\mathrm{M}_{2}$ and $\mathrm{M}_{3}\left(i_{D}^{M_{2}}\right.$ and $\left.i_{D}^{M_{3}}\right)$ are directed out of $\mathrm{R}_{\text {load. }}$ This suggests that the distortion due to $\mathrm{M}_{1}$ can cancel the distortion generated by $\mathrm{M}_{2}$ and $\mathrm{M}_{3}$. Using the weakly nonlinear distortion analysis approach in [5], for a certain attenuation value $0 \leq A \leq 1$, the IM3 output voltage is given by $\quad v_{\text {out }}^{\omega_{I M 3}} \propto \frac{3 \times V_{\text {in }}{ }^{3}}{(1+A)^{4} \cdot 16 R_{s}{ }^{3}} \times\left[\frac{16 A^{4}}{W_{M_{1}}{ }^{3}}-\frac{(1-A)^{4} A^{3}}{W_{M_{2}}{ }^{3}}-\frac{(1-A)^{4} A}{W_{M_{3}}{ }^{3}}\right]$

, where $\mathrm{W}$ is the transistor width. In deriving (1) the transistors' 3rd-order output conductance nonlinearity is assumed to dominantly contribute to IM3. Eq. (1) suggests that in the $\Pi$ attenuator, the IM3 current of $M_{1}$ at least partially cancels the IM3 current of $M_{2}$ and $M_{3}$ at the 
output. When $\frac{16 A^{3}}{W_{M_{1}}{ }^{3}}=\frac{(1-A)^{4} A^{2}}{W_{M_{2}}{ }^{3}}+\frac{(1-A)^{4}}{W_{M_{3}}{ }^{3}}$ there is full IM3 cancellation within the $\Pi$ attenuator, which is robust since it only relies on the ratio of transistor widths; then IIP3 is limited by mechanisms such as capacitive nonlinearity.

To verify the concept of (1) we implemented the $\Pi$ attenuator shown in Fig. 4.3.1(lower) with four parallel branches in a standard 0.16um CMOS process. For this attenuator, each parallel branch is designed for $-12 \mathrm{~dB}$ attenuation $(A=0.25)$ and has different width for $\mathrm{M}_{1}$ that yields either full or partial IM3 cancellation. For the measurements, we enable $M_{2}$ and $M_{3}$ while selectively enabling one parallel branch at a time. This mimics a $\Pi$ attenuator with a selectable $\mathrm{W}_{\mathrm{M} 1}$ for fixed $\mathrm{W}_{\mathrm{M} 2}(23 \mathrm{um})$ and $\mathrm{W}_{\mathrm{M} 3}(20 \mathrm{um})$. For a two-tone input signal centered at $1 \mathrm{GHz}$ with 3.2MHz spacing, the IIP3 is extrapolated from $-15 \mathrm{dBm}$ to $-10 \mathrm{dBm}$.

The measured and simulated IIP3 (using PSP model) as a function of $\mathrm{W}_{\mathrm{M} 1}$ is shown in Fig. 4.3.2, which verifies this IM3 cancellation theory. Note that the calculated optimal $\mathrm{W}_{\mathrm{M} 1}$ is $18 \mathrm{um}$ while it is 20um from simulations. For small $\mathrm{W}_{\mathrm{M} 1}, \mathrm{M}_{1}$ is dominant for the IM3. As $\mathrm{W}_{\mathrm{M} 1}$ increases its distortion decreases and hence IIP3 increases until its maximum at full IM3 cancellation. For even larger $\mathrm{W}_{\mathrm{M} 1}$ the IIP3 is dominated by $\mathrm{M}_{2}$ and $\mathrm{M}_{3}$ yielding a saturated sub-optimum value because $\mathrm{W}_{\mathrm{M} 2}$ and $\mathrm{W}_{\mathrm{M} 3}$ is fixed. Fig. 4.3.2 also shows the measured IIP3 for four different $\mathrm{W}_{\mathrm{M} 1}$ by sweeping the center frequency from $0.1 \mathrm{GHz}$ to $3 \mathrm{GHz}$. The difference between measured and simulated IIP3 are due to limited accuracy of transistor modeling. For the $\Pi$ attenuator with full IM3 cancellation $\left(\mathrm{W}_{\mathrm{M} 1}=20 \mathrm{um}\right)$, the IIP3 decreases as the $f_{\mathrm{rf}}$ increases because of the phase difference between the distortion current of $\mathrm{M}_{1}$ and that of $\mathrm{M}_{2}$ and $\mathrm{M}_{3}$ increasingly deviates from $180^{\circ}$ due to parasitic capacitances. As a result, the IM3 cancellation degrades as the frequency 
increases. Since this IM3 cancellation technique involves no extra devices but only properly dimensioning the transistors' $\mathrm{W}$, there is no need to trade large transistor for high linearity as in [3].

A four-branch attenuator system with selectable attenuation, see Fig. 4.3.3, using this IM3 cancellation technique was fabricated in a standard 0.16um CMOS process. Each branch contains one $\Pi$ attenuator that uses the topology shown in Fig. 4.3 .1 (upper) and is designed for one specific attenuation setting $(-6 \mathrm{~dB},-12 \mathrm{~dB},-18 \mathrm{~dB}$ and $-24 \mathrm{~dB})$ and optimized for full IM3 cancellation (transistor W fixed by (1)). A 3-to-8 digital decoder provides the controlling voltage (1.8V for enabling and $0 \mathrm{~V}$ for disabling). The active area of the attenuator and the decoder is $50 \times 30 u^{2}$ and $60 \times 65 u^{2}$ respectively. For each of the forenamed attenuation settings, only one branch is enabled. For minimum signal attenuation, the series transistors in all four branches are enabled, and the shunt transistors in all four branches are disabled, yielding an additional $-1.8 \mathrm{~dB}$ attenuation. For isolation and ac-bootstrapping purposes, the gate of transistor $M_{1}$ in the $\Pi$ attenuators is connected to the controlling voltage via a $20 \mathrm{k} \Omega$ resistor; while the bulk is connected to GND via a $20 \mathrm{k} \Omega$ resistor. The attenuator (see Fig. 4.3.7) was measured by on-wafer probing. The two-tone spacing is $3.2 \mathrm{MHz}$ for all measurements.

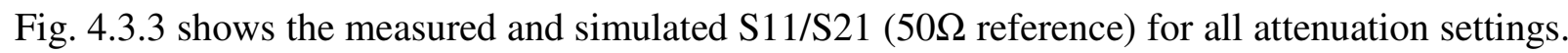
Due to a mistake in the decoder design, the minimum attenuation setting $(-1.8 \mathrm{~dB})$ cannot be enabled. For this setting we only show the simulated S11/S21. Due to unaccounted parasitics, the measured $\mathrm{S} 21$ for $f_{\mathrm{r}}>5 \mathrm{GHz}$ deviates $>1.6 \mathrm{~dB}$ from simulation. Since the proposed IM3 cancellation does not require large transistors, $5 \mathrm{GHz}$ bandwidth is achieved with $\mathrm{S} 11<-14 \mathrm{~dB}$ and 
with S21 variation $<1.6 \mathrm{~dB}$. The difference between the measured NF and measured insertion loss $(|\mathrm{S} 21|)$ is within $0.1 \mathrm{~dB}$ up to $10 \mathrm{GHz}$, which shows little noise is introduced by the attenuator.

Fig. 4.3.4 shows the measured HD1 and IM3 output at $1 \mathrm{GHz}$. The compression/expansion $\mathrm{P}_{1 \mathrm{~dB}}$ is $>10 \mathrm{dBm}$ for all attenuation settings. The IIP3 are respectively $31 \mathrm{dBm}, 33 \mathrm{dBm}, 38 \mathrm{dBm}$ and $36 \mathrm{dBm}$ for attenuation settings $-6 \mathrm{~dB},-12 \mathrm{~dB},-18 \mathrm{~dB}$ and $-24 \mathrm{~dB}$. Due to higher-order nonlinearities, the IM3 curves start to show $5^{\text {th }}$ order behavior for input power levels higher than $-8 \mathrm{dBm}$.

Fig. 4.3.5 shows the measured IIP3 by sweeping the input frequency $f_{\mathrm{rf}}$, from $0.1 \mathrm{GHz}$ to $10 \mathrm{GHz}$. We achieve $+30 \mathrm{dBm}$ IIP3 for the TV band (DC-1.2GHz) and $+26 \mathrm{dBm}$ for DC-5GHz. At higher $f_{\mathrm{rf}}$, extra phase shifts caused by the parasitic capacitances degrades the IM3 cancellation. The measured IIP3 of ten dies for $f_{\mathrm{rf}}=1 \mathrm{GHz}$ is also shown in Fig. 4.3.5. The IIP3 variation is within $1 \mathrm{~dB}$, which shows good robustness of this IM3 cancellation technique.

The benchmarking results in Fig. 4.3.6 shows that the presented attenuator achieves very high linearity for a very low active area in standard bulk CMOS. In conclusion this IM3 cancellation provides a robust IIP3 improvement with minimum active area consumption.

\section{Acknowledgements:}

We thank NXP for chip fabrication, and G. van der Weide, M. C. M. Soer and H. de Vries for assistance. 
References:

[1] Y.Y. Huang, W. Woo, Y. Yoon and C.H. Lee Y, "Highly linear RF CMOS variable attenuators with adaptive body biasing”, IEEE J. Solid-State Circuits, Vol. 46, No. 5, May 2011. [2] A.Youssef, J.Haslett and E.Youssoufian, "Digitally-controlled RF passive attenuator in 65 nm CMOS for mobile TV tuner ICs," in Proc. IEEE ISCAS, pp.1999-2002, 2010.

[3] M. Granger-Jones, et al., "A broadband high dynamic range voltage controlled attenuator MMIC with IIP3>+47 dBm over entire 30dB analog control range", in Proc. IEEE Microwave symposium Dig. (MTT), pp. 1-4, 2011.

[4] available in www.rfmd.com/CS/Documents/RFSA2013.pdf.

[5] W. Cheng, et al., "A general weak nonlinearity model for LNAs," in Proc. IEEE CICC, 2008.

[6] H.Dogan, et al., "Analysis and design of RF CMOS attenuators," IEEE JSSC No. 10, 2008.

[7] B. Ku, et al., "6-bit CMOS digital attenuators with low phase variations for X-band phasedarray systems," IEEE Tran. Microwave Theory and Techniques, Vol. 58, No. 7, July 2010. 
Figure captions:

Figure 4.3.1 Concept of the wideband IM3 cancellation for $\Pi$ attenuator and demonstration circuit for this concept.

Figure 4.3.2 Measured and simulated IIP3 illustrating the IM3 cancellation technique for the $\Pi$ attenuator. Symbol for measurement results, line for simulation results.

Figure 4.3.3 S11/S21 for the four-step attenuator. Line for measurement results, symbol for simulation results.

Figure 4.3.4 Measured output HD1 and IM3 vs input power for two tone input signals centered at $1 \mathrm{GHz}$ with $3.2 \mathrm{MHz}$ spacing.

Figure 4.3.5 Measured IIP3 as a function of the two tone center frequency $f_{\mathrm{rf}}$, with $3.2 \mathrm{MHz}$ frequency spacing and measured IIP3 of ten samples for $f_{\mathrm{rf}}=1 \mathrm{GHz}$.

Figure 4.3.6 Comparison of state-of-art attenuators.

Figure 4.3.7 Micrograph of the chip fabricated in a standard 0.16um bulk CMOS. 

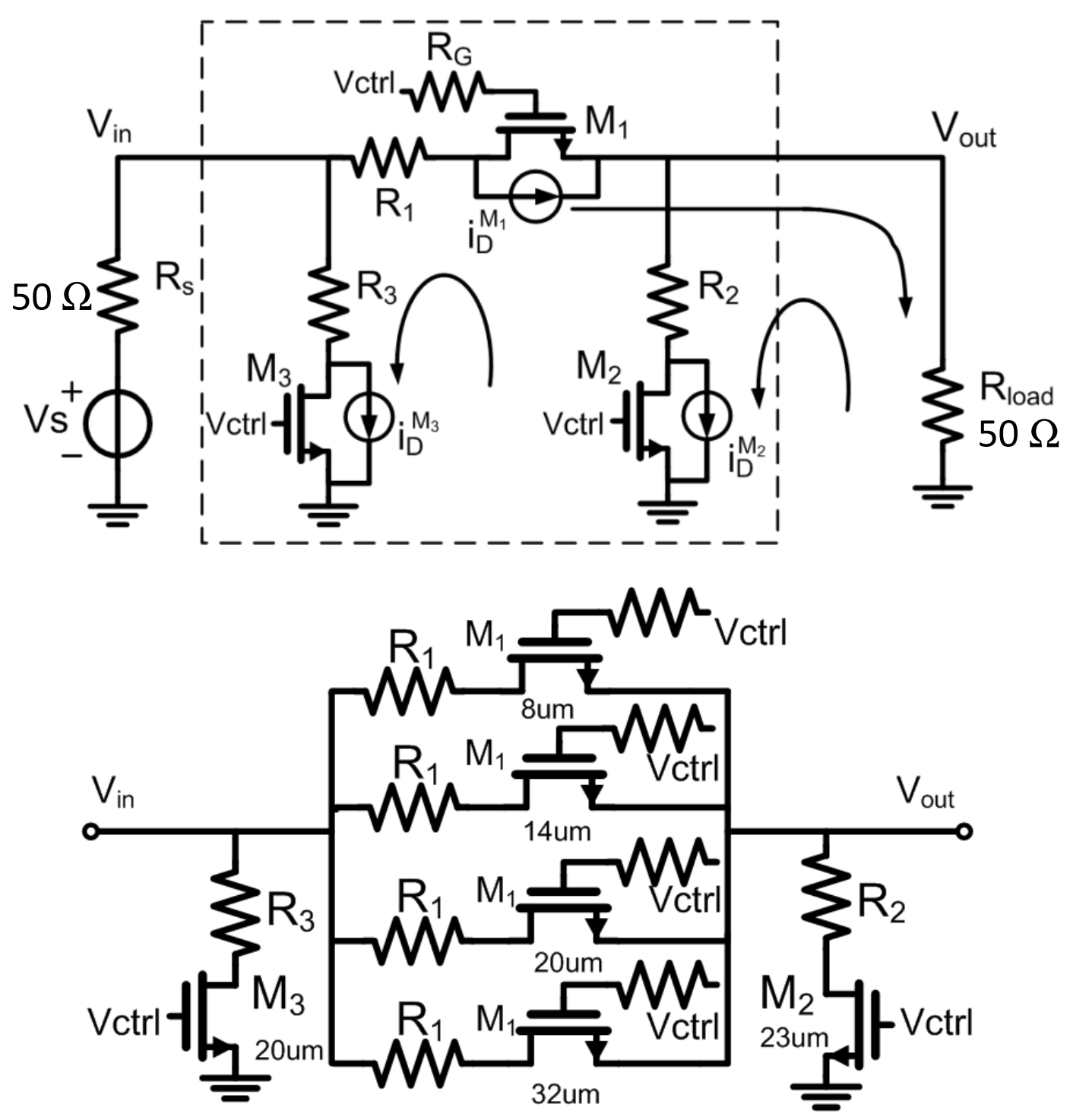

Figure 4.3.1: Concept of the wideband IM3 cancellation for $\Pi$ attenuator and demonstration circuit for this concept. 

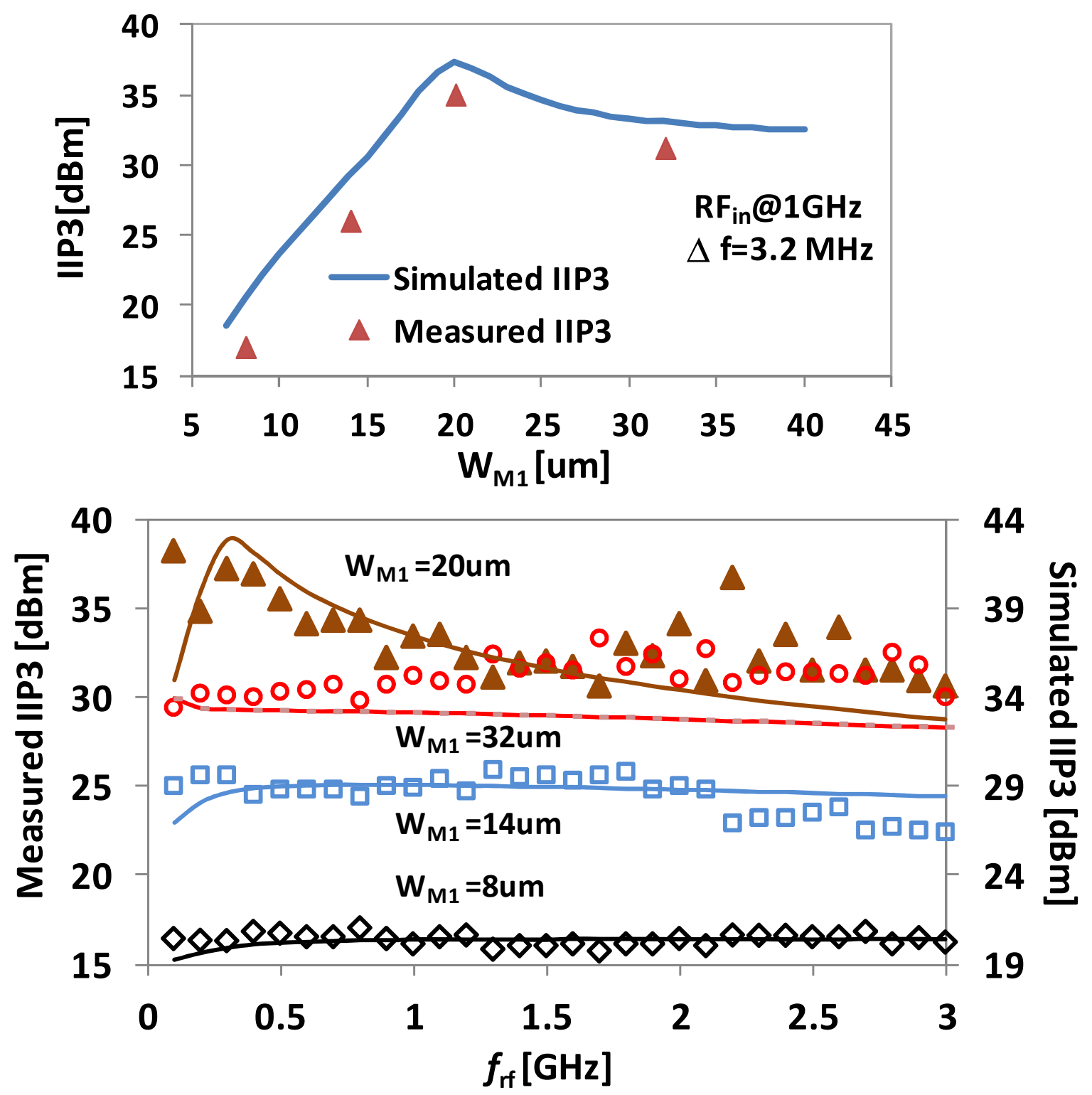

Figure 4.3.2: Measured and simulated IIP3 illustrating the IM3 cancellation technique for the $\Pi$ attenuator. Symbol for measurement results, line for simulation results. 

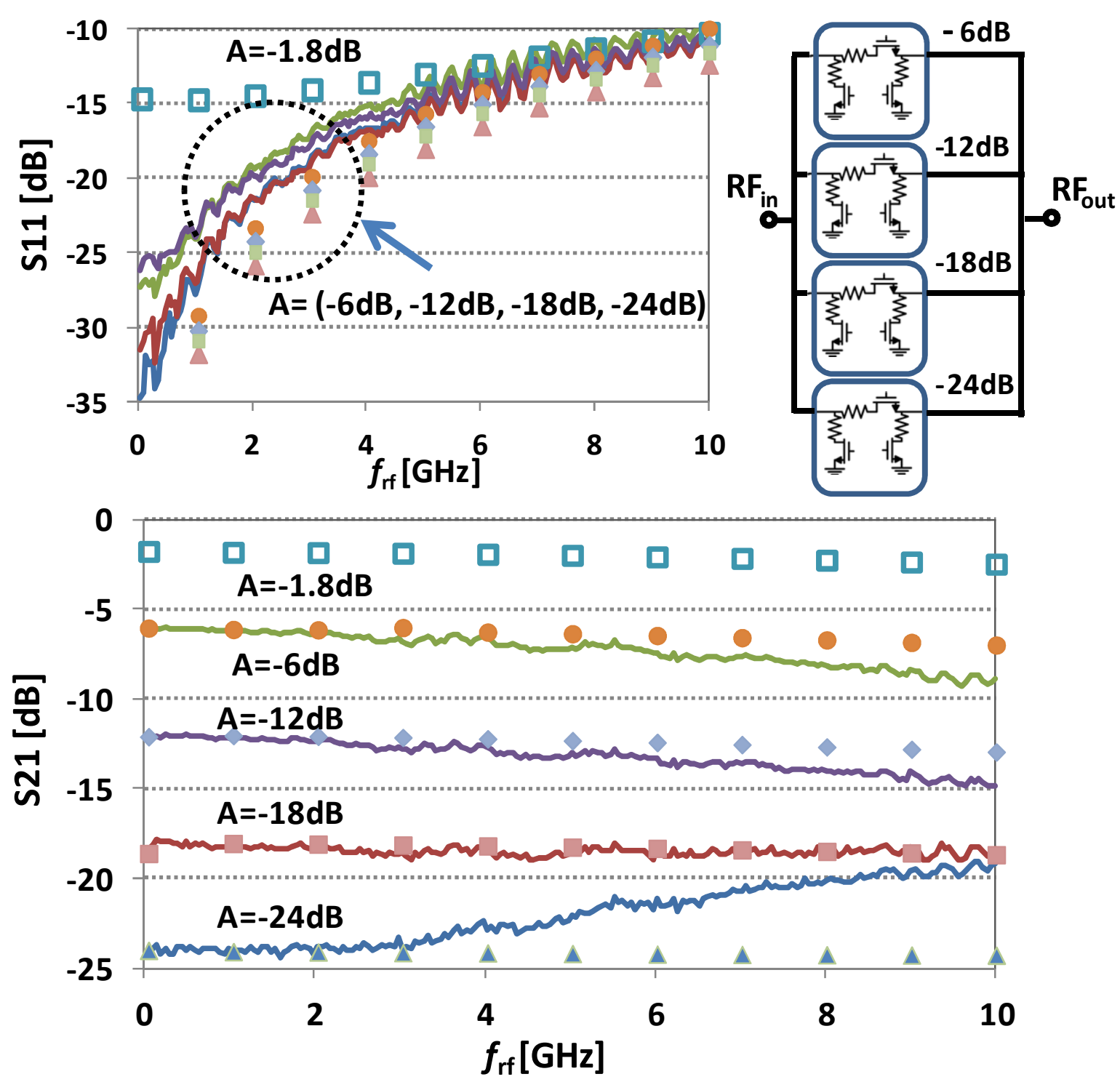

Figure 4.3.3: S11/S21 for the four-step attenuator. Line for measurement results, symbol for simulation results. 

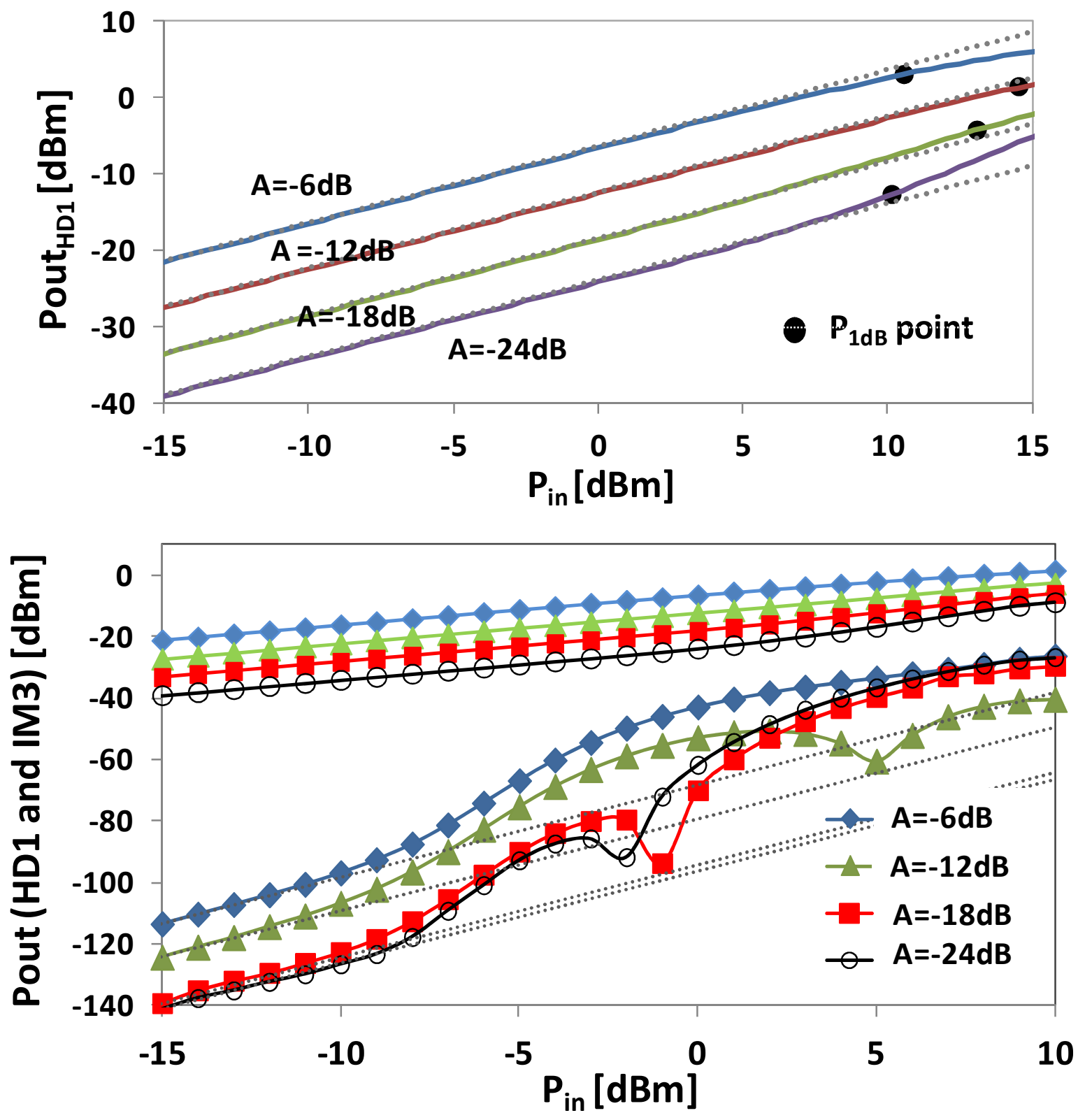

Figure 4.3.4: Measured output HD1 and IM3 vs input power for two tone input signals centered at $1 \mathrm{GHz}$ with $3.2 \mathrm{MHz}$ spacing. 

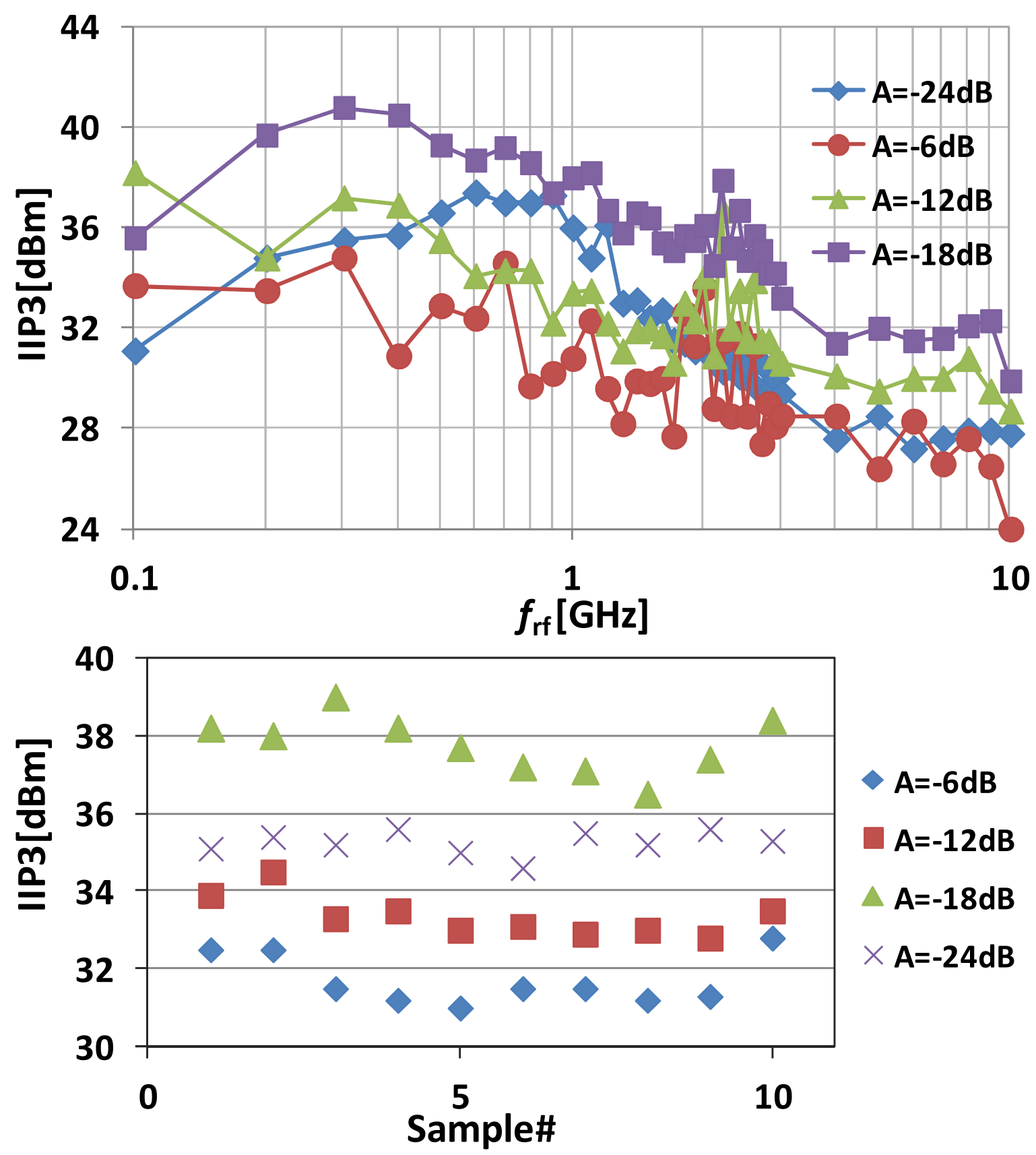

Figure 4.3.5: Measured IIP3 as a function of the two tone center frequency $f_{\mathrm{rf}}$, with 3.2 MHz frequency spacing and measured IIP3 of ten samples for $f_{\mathrm{rf}}=1 \mathrm{GHz}$. 


\begin{tabular}{|c|c|c|c|c|c|c|}
\hline & $\begin{array}{c}\text { Huang } \\
{[1]}\end{array}$ & $\begin{array}{c}\text { Youssef } \\
{[2]}\end{array}$ & $\begin{array}{c}\text { Dogan } \\
{[6]}\end{array}$ & $\begin{array}{c}\text { Granger- } \\
\text { Jones[3] }\end{array}$ & Ku[7] & This work \\
\hline Technology & $\begin{array}{c}0.18 \mathrm{um} \\
\text { CMOS }\end{array}$ & $\begin{array}{c}65 \mathrm{~nm} \\
\text { CMOS }\end{array}$ & $\begin{array}{c}0.13 \mathrm{um} \\
\text { CMOS }\end{array}$ & $\begin{array}{c}\text { CMOS } \\
\text { SOI }\end{array}$ & $\begin{array}{c}0.18 \mathrm{um} \\
\text { CMOS }\end{array}$ & 0.16 um CMOS \\
\hline VDD [V] & 1.8 & 1.2 & 1.2 & 5 & N/A & 1.8 \\
\hline Chip area[mm $\left.{ }^{2}\right]$ & 0.28 & 0.05 & 0.7 & N/A & 0.5 & 0.0054 \\
\hline Bandwidth[GHz] & $0.4-3.7$ & $0.4-0.8$ & DC-2.5 & $0.05-4$ & DC-14 & DC-5 \\
\hline IIP3[dBm] & +15 & +23 & +10 & +47 & $29 @ 10 \mathrm{GHz}$ & $\begin{array}{c}+30(0.1-1.2 \mathrm{GHz}) \\
+26(0.1-5 \mathrm{GHz})\end{array}$ \\
\hline P1dB[dBm] & +6 & N/A & +2.5 & 30 & $15 @ 10 \mathrm{GHz}$ & +10 \\
\hline $\begin{array}{c}\text { Attenuation } \\
\text { flatness[dB] }\end{array}$ & 2.6 & N/A & 2.6 & 3 & 0.5 & 1.6 \\
\hline $\begin{array}{c}\text { Max. } \\
\text { attenuation[dB] }\end{array}$ & 33 & 48 & 42 & 40 & 31.5 & 24 \\
\hline $\begin{array}{c}\text { Minium } \\
\text { attenuation[dB] }\end{array}$ & $0.96-2.9$ & N/A & $0.9-3.5$ & $2.4-4$ & $3.7-10$ & $1.8-2.4($ sim) $)$ \\
\hline Return loss[dB] & $>9$ & $>12$ & $>8.2$ & $>14$ & $>9$ & $>14$ \\
\hline Control mode & $\begin{array}{c}\text { Linear- } \\
\text { in-dB }\end{array}$ & $\begin{array}{c}\text { Discrete } \\
\text { step }\end{array}$ & $\begin{array}{c}\text { Linear-in- } \\
\mathrm{dB}\end{array}$ & $\begin{array}{c}\text { Linear-in- } \\
\mathrm{dB}\end{array}$ & $\begin{array}{c}\text { Discrete } \\
\text { step }\end{array}$ & Discrete step \\
\hline
\end{tabular}

Figure 4.3.6: Comparison of state-of-art attenuators. 


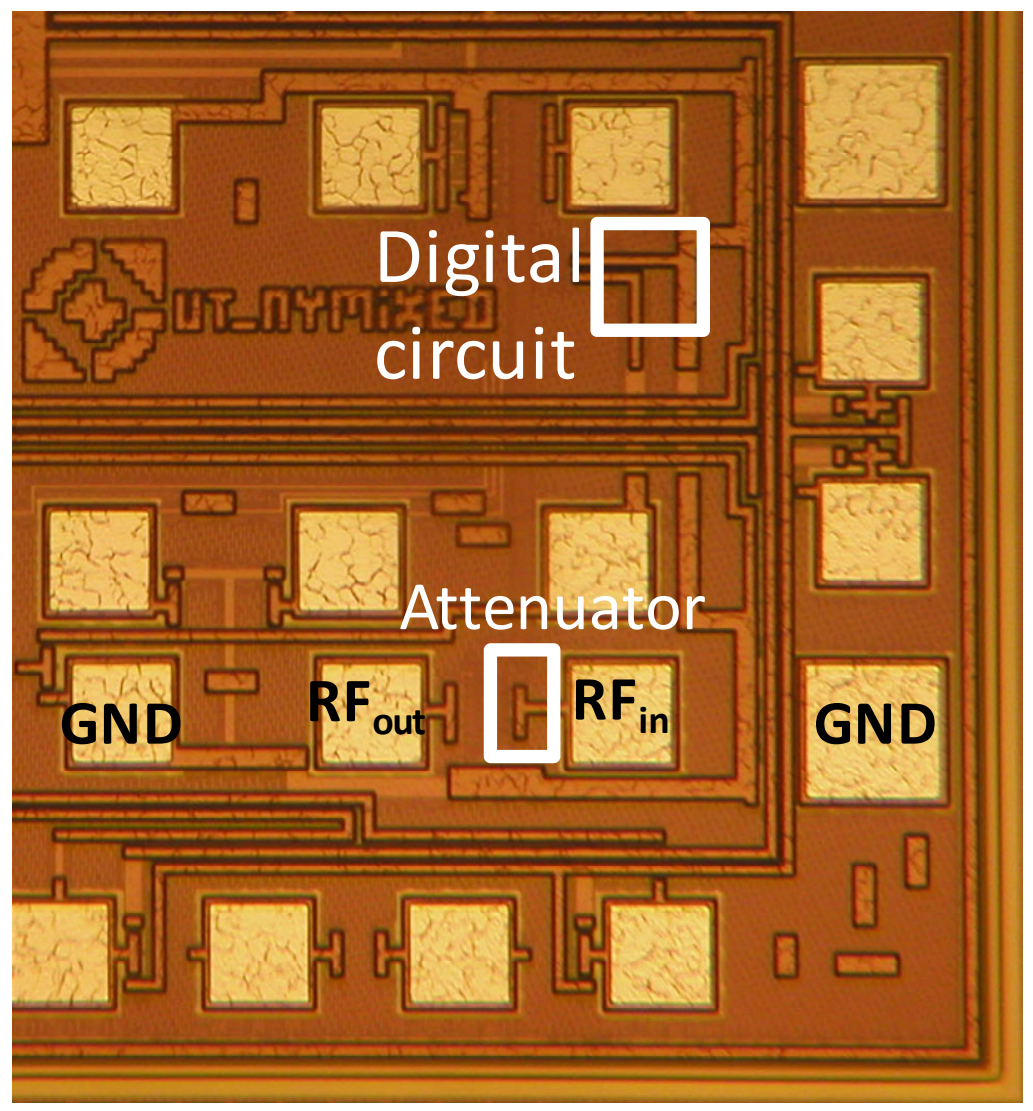

Figure 4.3.7: Micrograph of the chip fabricated in a standard 0.16um bulk CMOS. 